\title{
Water and Life: The Medium is the Message
}

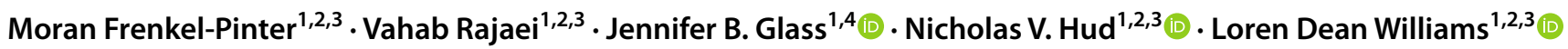

Received: 23 August 2020 / Accepted: 21 November 2020 / Published online: 11 January 2021

(c) The Author(s) 2021

\begin{abstract}
Water, the most abundant compound on the surface of the Earth and probably in the universe, is the medium of biology, but is much more than that. Water is the most frequent actor in the chemistry of metabolism. Our quantitation here reveals that water accounts for $99.4 \%$ of metabolites in Escherichia coli by molar concentration. Between a third and a half of known biochemical reactions involve consumption or production of water. We calculated the chemical flux of water and observed that in the life of a cell, a given water molecule frequently and repeatedly serves as a reaction substrate, intermediate, cofactor, and product. Our results show that as an E. coli cell replicates in the presence of molecular oxygen, an average in vivo water molecule is chemically transformed or is mechanistically involved in catalysis $\sim 3.7$ times. We conclude that, for biological water, there is no distinction between medium and chemical participant. Chemical transformations of water provide a basis for understanding not only extant biochemistry, but the origins of life. Because the chemistry of water dominates metabolism and also drives biological synthesis and degradation, it seems likely that metabolism co-evolved with biopolymers, which helps to reconcile polymer-first versus metabolism-first theories for the origins of life.
\end{abstract}

Keywords Metabolism $\cdot$ Metabolite $\cdot$ Glycolysis $\cdot$ Amino acid $\cdot$ Translation $\cdot$ Oxidative phosphorylation

\section{Introduction}

Water is the most abundant liquid on the surface of the Earth (Weingärtner et al. 2000) and life as we know it demands water (Brack 1993; Ball 2017). Biochemist Albert SzentGyörgyi stated: "Water is life's matter and matrix, mother and medium. There is no life without water" (Szent-Györgyi

Handling editor: Ulrich Muller.

Electronic supplementary material The online version of this article (https://doi.org/10.1007/s00239-020-09978-6) contains supplementary material, which is available to authorized users.

Loren Dean Williams

loren.williams@chemistry.gatech.edu

NASA Center for the Origins of Life, Atlanta, GA, USA

2 NSF-NASA Center of Chemical Evolution, Atlanta, GA, USA

3 School of Chemistry and Biochemistry, Georgia Institute of Technology, 315 Ferst Drive NW, Atlanta, GA 30332-0400, USA

4 School of Earth and Atmospheric Science, Georgia Institute of Technology, 311 Ferst Drive NW, Atlanta, GA 30332-0340, USA
1971). Most cells are around 65\% water by volume and $70 \%$ by mass (Milo and Phillips 2015), and living organisms have developed sophisticated mechanisms for appropriating and conserving water (Milo and Phillips 2015). The properties of water, and its roles as the biological medium, have been extensively characterized. Water drives folding and assembly of biopolymers (Kauzmann 1959; Radzicka et al. 1988; Sundaralingam and Sekharudu 1989; Barron et al. 1997) and supports the formation of membranes and other biological structures. Similarly, it is well documented that water is produced, consumed, altered, or utilized transiently during enzymatic reactions (Testa and Kraemer 2007; Nagano et al. 2015). Water is a metabolite (Kim et al. 2007), which is defined as an intermediate or end product of metabolic reactions (Venes 1940), even though it is usually not described as such (for example, see Fell and Wagner 2000; Testa and Kraemer 2007; Bennett et al. 2009; Moradi et al. 2017).

Water defines the chemistry of biology and is the dominant chemical actor in the connected chemical reactions that maintain the cellular living state, i.e., in metabolism. Water is chemically involved in a plurality of biochemical transformations. The ability to chemically consume and produce water is the unifying theme of organic and inorganic molecules of biology. Essentially all biological molecules, 
large and small, are products of or substrates for biochemical reactions that transform water.

A survey here suggests that one third to one half of known enzymatic reactions consume or produce water. This fraction under-represents involvement of water in enzymology because it omits reactions that use water transiently during catalysis. A second survey tallied the number of water molecules involved in two dominant cellular metabolic hubs: protein synthesis and oxidative phosphorylation. The results show that an average water molecule is chemically transformed or is mechanistically involved in catalysis about 3.7 times by these two processes as an Escherichia coli (E. coli) replicates in the presence of molecular oxygen $\left(\mathrm{O}_{2}\right)$. Based on these quantitative data, we define water as the chemical cornerstone of biological processes and challenge the notion or even the possibility of an inert biological solvent.

Notably, our quantitative demonstration of water as the dominant reactive species in biology is distinct from previously defined "metabolic waters" (Mellanby 1942). That term focuses on water molecules produced by specific metabolic processes and excludes many other transformations of water in biochemical processes, such as water molecules involved (i) in hydrolysis, (ii) as intermediates, or (iii) mechanistically in enzymes.

\section{Results}

\section{Reactive Core of Biology: Defining Transformations of Water}

Metabolism is a complex network of chemical reactions that are kinetically and thermodynamically linked by common substrates, intermediates, products, and effectors. Water is a key agent of cooperation of a core set of metabolic reactions. The production, consumption, and catalytic utilization of water in the citric acid cycle are illustrated (Fig. 1). Reactions that transform water include (i) condensation dehydration and intramolecular dehydration reactions, that chemically produce water; (ii) hydrolysis reactions, that chemically consume water; (iii) reactions that use water mechanistically during catalysis; (iv) reactions that produce protons, hydroxide, carbon dioxide, peroxide, etc., which cause downstream chemical reactions of water; (v) reactions that split water (e.g., in photosynthesis); and (vi) changes in metal coordination that release or absorb water.

Many water-consuming reactions are catalyzed by the hydrolase family of enzymes and involve cleavage of pyrophosphates, esters, peptides, acetyls, ethers, etc. (Supporting Fig. S1). Water is also consumed by many oxidoreductase, lyase, and transferase enzymatic reactions. Some water-consuming reactions are enabled by acid-base chemistry: chemical transformation of water molecules into active catalysts via protonation of water to form hydronium ions (allowing acid catalysis) and deprotonation to form hydroxide ions (allowing base catalysis; Supporting Fig. S1c) (Saá and Frontera 2020). Production of water in chemical reactions is common in chemical linkage and dehydration reactions; examples include linkages between and among acids, alcohols, amines, aldehydes or ketones, to form esters, peptides, or acetals (Supporting Fig. S1g). Changes in metal coordination chemically take up or release water. For example, when the ribosome assembles, rRNA coordinates $\mathrm{Mg}^{2+}$ ions and releases water from the first hydration shell of the metal (Bowman et al. 2012) (Supporting Fig. S1f). Release of water from divalent metal coordination is a form of chemical transformation (Capaldi and Aggeler 2002).

An example of the extensive involvement of water transformations in biochemistry is demonstrated in Fig. 1, which is a water-centric representation of the citric acid cycle (also termed the Krebs cycle). Except for the conversion of succinyl-CoA to succinate, water molecules chemically participate in each step of the citric acid cycle.

Of 172 metabolites listed in the Microbial Metabolome Database (Wishart et al. 2018), 163 contain at least 1 functional group capable of condensation dehydration and/ or hydrolysis reactions. The central metabolite coenzyme A illustrates how deeply and completely the chemistry of water is integrated into metabolic processes. Coenzyme A can be hydrolyzed to form adenine, ribose, phosphate, pyrophosphate, $\beta$-alanine, $\beta$-mercaptoethylamine, and pantoic acid. Several hydroxyl groups, a thiol, and a phosphate of coenzyme A are available for condensation dehydration reactions.

\section{The Enzyme Commission Database}

The Enzyme Commission (Apweiler et al. 2010) (EC) classifies biochemical reactions by products and reactants, independent of enzyme mechanism or structure. A survey here indicated that between a third and a half (2739 out of 6520) of EC reactions contain water as either a substrate or a product (Fig. 2; Table S1). By contrast, the EC database contains fewer than 1000 reactions that consume or produce ATP and fewer than 460 reactions that consume or produce NAD ${ }^{+}$. The importance of water chemistry is also seen in alternative enzyme reaction classifications, such as those that classify reactions based on reactive site structures and catalytic mechanism. For example, approximately a half (445/879) of enzymatic reactions in the EzCatDB database (Nagano et al. 2015) consume or produce water.

\section{Quantitating Transformations of Water in E. coli}

We calculated a lower limit for frequency of participation of water molecules in chemical transformation during 
Fig. 1 Chemical transformations of water by the citric acid cycle. Eight enzymes (green text) catalyze a series of reactions that in total, consume three water molecules, produce one water molecule, protonate three water molecules, and convert an acetyl group to two carbon dioxide molecules. The series of reactions produces three $\mathrm{NADH}$, an $\mathrm{FADH}_{2}$, and a GTP. Unprotonated water molecules are indicated by red spheres. Protonated water molecules are blue. Water molecules that are mechanistically involved in the reactions are green (Color figure online)

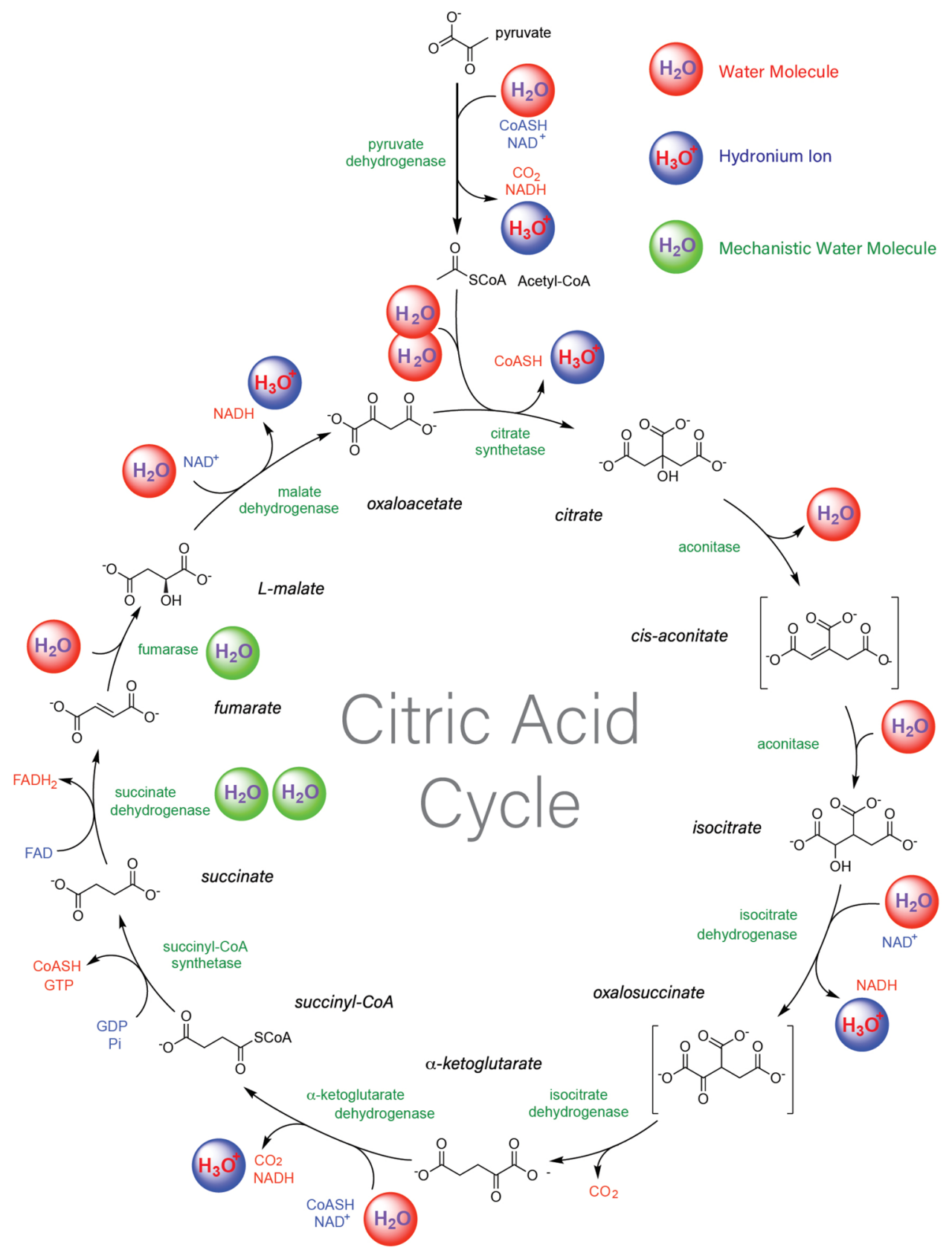

replication of a single E. coli. We focused on protein production and oxidative phosphorylation with the assumption of oxic conditions in minimal medium. The estimate of the quantity of water transformed is a lower limit because it does not account for many water molecules used mechanistically during catalysis, those released or taken up by changes in metal coordination, those used in downstream chemical reactions of $\mathrm{CO}_{2}$ or $\mathrm{H}_{2} \mathrm{O}_{2}$, nor those chemically transformed outside of protein synthesis and oxidative phosphorylation. The focus was on protein synthesis and oxidative phosphorylation because they are dominant metabolic hubs (Russell and Cook 1995; Buttgereit and Brand 1995), requiring immense chemical energy and participation of many metabolites. Protein comprises approximately half of the dry weight of an E. coli (Milo 2013; Milo and Phillips 2015) and protein synthesis is a major metabolic consumer in biological systems (Stouthamer 1973). Biochemical reaction pathways and reaction mechanisms were inspected as outlined in detail in the "Materials and Methods" section. We tallied the number of water molecules that chemically participate in each step of these reaction pathways and counted the number of water molecules consumed or produced. Wherever possible, we identified water molecules involved mechanistically in catalytic function or as intermediates. 

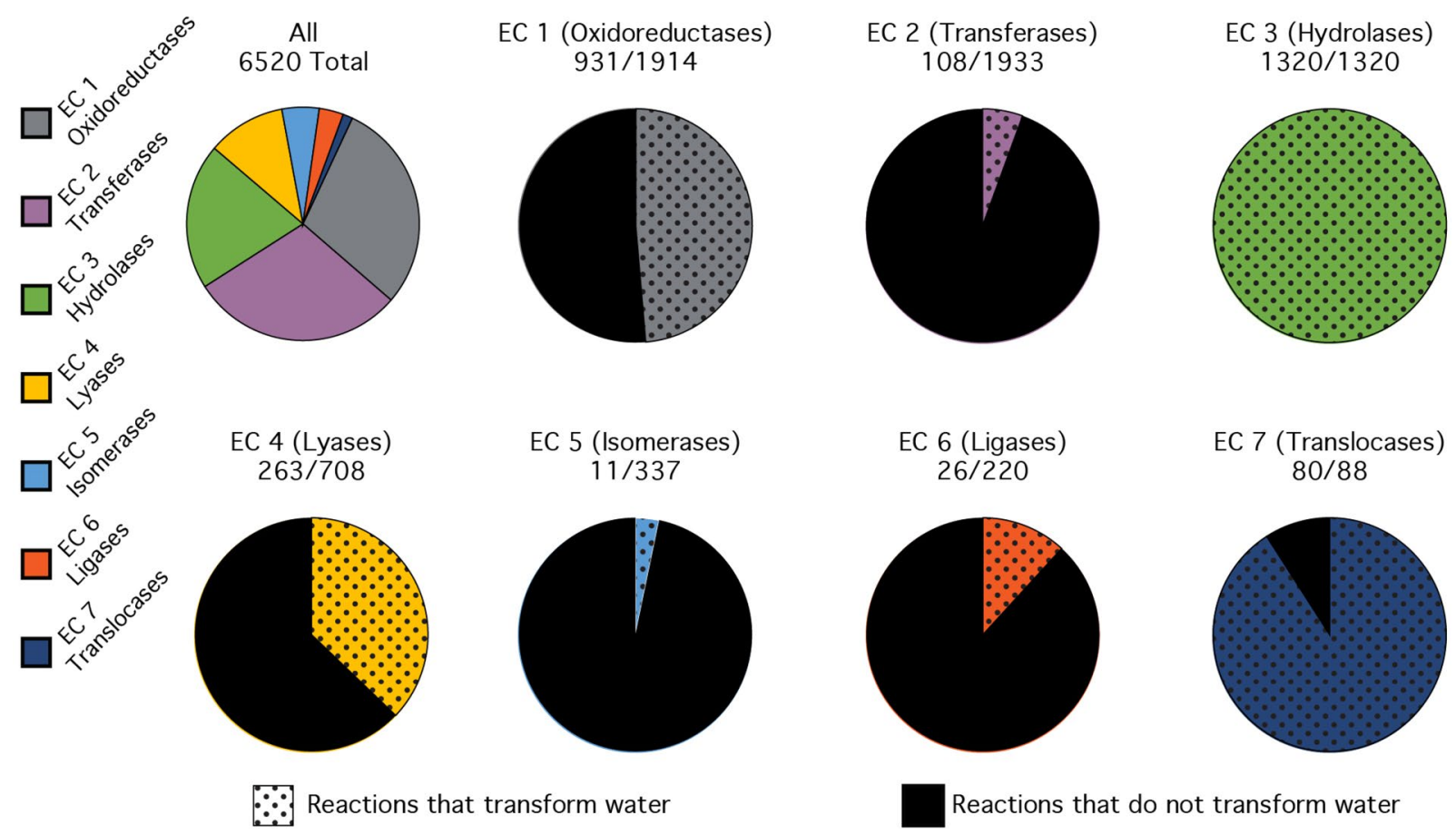

EC 7 (Translocases) $80 / 88$

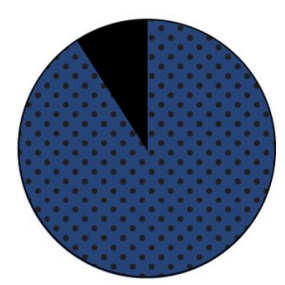

Reactions that do not transform water

Fig. 2 Many biological reactions involve transformations of water. A total of $42 \%$ (2739/6520) of Enzyme Commission (EC) reactions use water as either a substrate or a product. The distribution of reactions

For protein synthesis, relevant reactions include those that produce amino acids from products of glycolysis and the citric acid cycle (Fig. 1; Supporting Figs. S3-S22), and those that polymerize amino acids to form proteins (see "Materials and Methods" section). Our results show that approximately $3.42 \times 10^{-14} \mathrm{~mol}$ of water $\left(6.16 \times 10^{-13} \mathrm{~g}\right)$ participate in chemical transformations related to amino acid biosynthesis and amino acid polymerization as E. coli replicates. An E. coli cell contains about $3.9 \times 10^{-14} \mathrm{~mol}$ of water. Therefore, about $88 \%$ of water molecules in an $E$. coli cell participate chemically in reactions of amino acid biosynthesis and polymerization.

E. coli living on glucose requires about $10 \times 10^{9} \mathrm{ATP}$ molecules $\left(1.66 \times 10^{-14} \mathrm{~mol}\right)$ to replicate (Farmer and Jones 1976; Phillips and Milo 2009). Many water molecules participate in the reactions of aerobic respiration, which produces about $1.45 \times 10^{-14} \mathrm{~mol}$ of ATP during replication of an E. coli cell in the presence of $\mathrm{O}_{2}$ (Farmer and Jones 1976; Phillips and Milo 2009). Approximately, 28 out of 32 ATPs generated from one glucose molecule are produced through oxidative phosphorylation (Demirel 2013; Sokolov et al. 2015). As part of the oxidative phosphorylation pathway, ATP synthase catalyzes dehydration of ADP and Pi into an ATP molecule. In addition, ATP synthase uses two mechanistic water molecules for each proton that is translocated across the membrane from the among seven EC classes is shown, along with the portion of watertransforming reactions in each class

periplasm to the cytoplasm (Capaldi and Aggeler 2002). Since 3.3 protons are required for the generation of 1 ATP molecule by ATP synthase (Capaldi and Aggeler 2002; Voet and Voet 2010; Hahn et al. 2016), and 2 water molecules are required mechanistically for the transport of each of those protons, a total of about 7 water molecules are involved in the generation of 1 ATP molecule by ATP synthase (including the dehydration of ADP and Pi into ATP). Moreover, during oxidative phosphorylation, there is delivery of electrons by the reduced NADH and $\mathrm{FADH}_{2}$ to $\mathrm{O}_{2}$. Dioxygen is split to form water. Taken together, we calculated that $1.1 \times 10^{-13} \mathrm{~mol}\left(2.0 \times 10^{-12} \mathrm{~g}\right)$ of water chemically participate in processes of oxidative phosphorylation during replication of an E. coli (see "Materials and Methods" section). The amount corresponds to $278 \%$ of the total number of water molecules in an E. coli cell, indicating that an average water molecule is repeatedly transformed.

Taken together, our results show that $1.4 \times 10^{-13} \mathrm{~mol}$ of water chemically participate in protein synthesis and oxidative phosphorylation during the replication of an $E$. coli. This calculation implies that in the lower limit, an average water molecule is chemically transformed or is mechanistically involved in catalysis at least 3.7 times as an E. coli replicates. Our results demonstrate that an average biological water molecule is used repeatedly in chemical reactions during the life of a cell. 


\section{Discussion}

\section{Biochemistry is Chemistry of Water}

The quantitative data show that water defines the chemistry of biology and is the dominant chemical actor in metabolism. Water accounts for $99.4 \%$ of metabolites in an $E$. coli by molar concentration (Fig. 3). The unifying theme of organic and inorganic molecules of biology is chemical production and/or consumption of water during their catabolism and anabolism. Polymer building blocks, polymers, and metabolites are chemically transformed by water. $42 \%$ of known enzymatic reactions chemically transform water (Fig. 2). For replication of an E. coli cell, the integrated flux of water through chemical processes appears to be greater for water than for any other molecule. Water is the reactive nexus of biology and the primary agent of chemical, thermodynamic, and kinetic linkage of biochemical processes.

A model of water as the reactive nexus of biology is qualitatively supported by the enormous number and diversity of enzymatic reaction types that produce or consume water and by the criticality of chemical transformations of water in reactions involving biopolymers, metals, and organic cofactors. The number of reaction types that transform water is far greater than the number of reaction types that transform other metabolites (such as ATP and $\mathrm{NAD}^{+}$). Chemical transformations of water are widespread in the core chemistry of life, including biosynthesis of amino acids, nucleotides, and membrane components (Voet and Voet 2010). Water is chemically transformed during information processing, as in the synthesis of DNA (Patel et al. 2011) and RNA (Belogurov and Artsimovitch 2015), and in synthesis of carbohydrates and phospholipids (Runnels et al. 2018). Transformations of water are also key to central metabolism, in glycolysis, the citric acid cycle, oxidative phosphorylation, and photosynthesis (Voet and Voet 2010).

A model of water as the reactive nexus of biology is quantitatively supported by the frequency of chemical transformations of water during replication of a single $E$. coli cell. The average water molecule participates repeatedly in these chemical transformations. Our quantification is a lower limit; we have not included waters used in processes such as synthesis of RNA, DNA, and membrane lipids. We did not count water molecules involved in changes in metal coordination. Moreover, mechanisms for many enzymes are not fully understood and might involve water molecules that were not accounted for here. Anaerobiosis is an important consideration; facultative anaerobes such as E. coli are capable of growth under both oxic and anoxic conditions (Tran and Unden 1998). Our quantitative results assume oxic conditions, whereas under anoxic conditions, anaerobic metabolic systems would transform water molecules in a pathway-dependent manner, to an extent that depends on availability of electron acceptors (such as nitrate and fumarate). Specifically, under anoxic conditions, if electron acceptors are available, E. coli would perform anaerobic respiration instead of fermentation; the former is expected to use a similar number of water molecules as aerobic respiration, mostly due to the heavily water-consuming, ATP synthase, which is common to both types of respiration pathways (Ingledew and Poole 1984). On the other hand, if fermentation is used as the major energy-producing pathway, fewer transformations of water molecules would occur because fermentation does not involve ATP synthesis through ATPase.

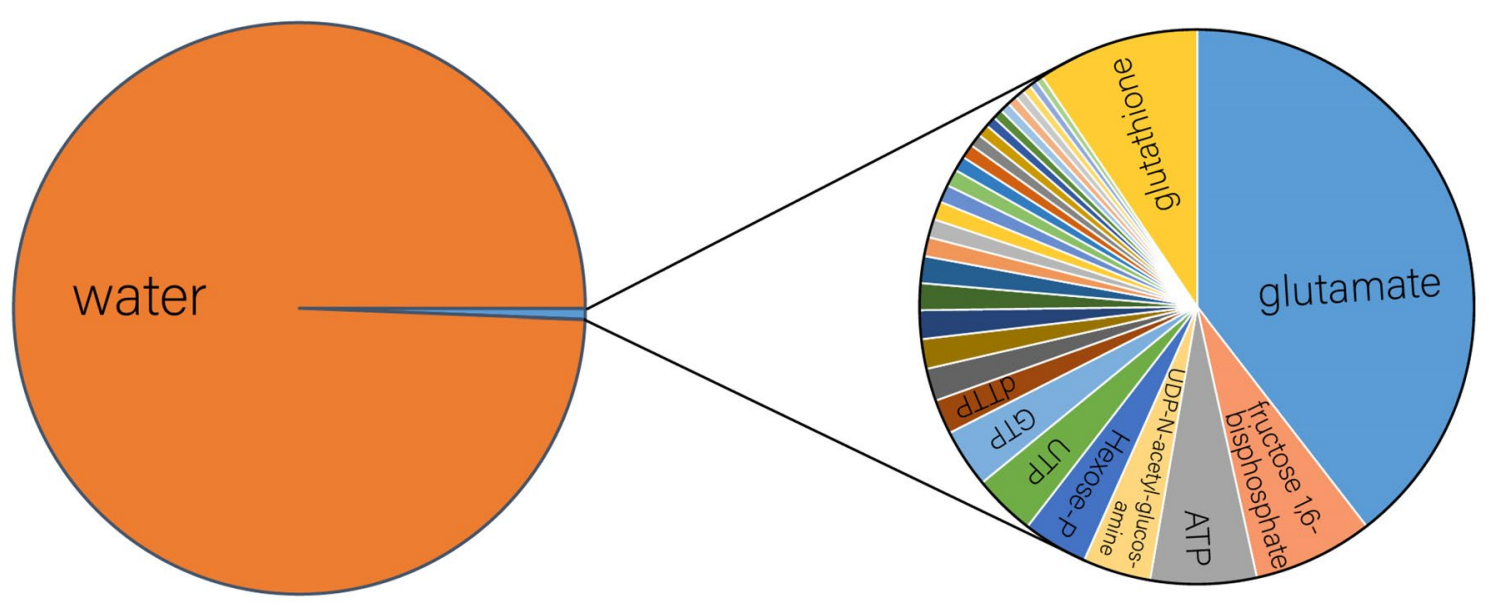

Fig. 3 Water is a dominant metabolite in biochemistry, accounting for $99.4 \%$ by molarity of metabolites within an E. coli. Water in an E. coli cell is around $40 \mathrm{M}$ (see "Materials and Methods" section). The sum of the concentrations of all other metabolites is $240 \mathrm{mM}$ 


\section{General Implications for Biology}

Water is (i) a unique medium that promotes complex molecular assemblies (Ball 2017), (ii) highly reactive (Brack 1993), (iii) the dominant metabolite, and (iv) an agent in chemical transformations of a great majority of biological molecules. Water forms a continuum from medium to chemical participant. By extent of chemical participation, water dominates the chemistry of biology at a fundamental level. The sharing of water molecules as a common substrate, intermediate, and/or product provides mechanisms of chemical, thermodynamic, and kinetic linkage across an enormous number of reactions. Incorporation of water as a metabolite into existing metabolite maps (such as the IUBMB-Nicholson Metabolic Maps) would reveal a dense network of associations. Broad analysis of water metabolism across various pathological conditions has not been established thus far and might reveal interesting water-related metabolic defects that could contribute to disease development.

A large number of water molecules are observed in X-ray structures, linking enzymes with substrates, intermediates, effectors or products. These water molecules beautifully complement enzyme-ligand complexes that encompass them. For example, many water molecules form specific hydrogen bonds that mediate interactions between nucleotide substrates and succinyl coenzyme A synthetase, an enzyme in the citric acid cycle that interconverts succinylCoA and succinate. Are these waters part of the medium or are they specific actors in a reaction mechanism? They are both, there is no distinction.

\section{The Origins of Life on Earth}

The centrality of water in biochemistry, both as a medium and in chemical processes, can help us understand prebiotic chemistry and the chemical origins of life. A simple explanation for what one observes in extant biochemistry is that prebiotic chemical selection, leading to life, was substantially directed by water. Water was a primary gatekeeper that allowed in or denied access to organic molecules and metals during prebiotic chemistry. Specifically, chemical evolution selected building blocks that (i) were soluble in water, (ii) were chemically transformed by water, (iii) chemically transformed water, (iv) joined covalently, by condensation-dehydration reactions with near-equilibrium thermodynamics with accessible kinetics. Building blocks that participated in water chemistry and were chemically linked via water chemistry were selected to form small chimeras (e.g., triglycerides, coenzyme A, or nucleotides) and large homogeneous polymers (DNA, RNA, protein, polysaccharide). Potential building blocks that did not participate in water chemistry were excluded. This scenario explains the exclusion of various molecules from extant biochemistry, such as polycyclic aromatic hydrocarbons, which are highly abundant in the abiotic universe (Hertzog et al. 2019; Li 2020), but do not react with water.

\section{Proto-Metabolism}

The activity of water on land surfaces of our rotating planet is in fluctuating disequilibrium, causing oscillations in directionalities of water-based chemical reactions. These geochemical phenomena are replicated by experimental "wet-dry chemistry" (Mamajanov et al. 2014; Forsythe et al. 2015; Ross and Deamer 2016; Frenkel-Pinter et al. 2019; Doran et al. 2019), which uses chemical transformations of water to build proto-biological oligomers. In these experiments, environmental energy is transduced to chemical energy by near-equilibrium, kinetically accessible reactions. Thus wet-dry chemistry can be considered a form of water-centric proto-metabolism.

Near-equilibrium, oscillating reactions driven by cycling water activity provide experimental platforms for chemical evolution, allowing the exploration of open-ended chemical change. Oligomers form and degrade; those that fold and assemble degrade more slowly, increase in relative population (Runnels et al. 2018). These experimental systems link proto-metabolism to biopolymer synthesis. It seems likely that metabolism co-evolved with polymers, neither was first.

\section{Summary}

The Turkish novelist Mehmet Murat ildan said, "Water is the most perfect traveler because when it travels it becomes the path itself." American poet Lucy Larcom said, "A drop of water, if it could write out its own history, would explain the universe to us." Water is transformative and is transformed, eternally causing change and constantly changing. Water is the gate-keeper and the matrix. This duality is seen from planets, during formation of oceans and tectonic plates, to cells, during energy transduction and biopolymer assembly. We suggest that intense chemical participation of the medium might be an important characteristic of living systems in general. It is possible that other liquids, such as ammonia at low temperatures, or organic eutectics, might meet these requirements.

The picture that emerges from this analysis is of enzymatic systems integrated with each other by chemical transformations of the medium. The unusual physical properties of water as a solvent are coupled with frequent participation in diverse chemical transformations during the life of a cell. Water is never absent or physically separated from biological macromolecules, organic cofactors, and metals, but readily combines with, withdraws from, and intercedes in their transformations. In biological systems, water is fully integrated into processes of bond making and bond breaking. 
The distinction between medium and chemical participant is so blurred as to be devoid of meaning. This quantitative demonstration of water as the dominant metabolite in biochemistry suggests, based on expectations of continuity in evolution, that origins of life studies should focus on scenarios where the chemistry of water is central to emergence of biopolymers, organic metabolites, and metabolism. Leonardo da Vinci was correct: water is the ultimate vehicle of nature.

\section{Materials and Methods}

\section{General Approach to Analysis of Water biochemistry}

To calculate the number of water molecules that participate in chemical transformation during replication of a single $E$. coli (a doubling event), we focused on protein synthesis and oxidative phosphorylation. These two processes involve the greatest pluralities of chemical transformations within a cell and present the greatest energy flux. We assume that replication of an E. coli requires reproduction of every chemical species in an E. coli, except those that are supplied by the media. We analyzed metabolic processes required to make these chemical species. Our results here are for an $E$. coli that is replicated on minimal media under aerobic condition. We presume standard M9 minimal media (Anonymous 2010). Changes in media and in oxygen availability will change the specific numbers here, but will not change the general conclusion that water is intimately involved in chemical processes in living systems and is a predominant metabolite in biochemistry.

The water molecules accounted for here, as chemical participants, are either directly consumed or produced in transformation of substrate into product, or are involved mechanistically in the catalytic activity of the enzymes as intermediates or as catalysts. The role of water molecules in enzyme mechanism is commonly uncharacterized. Therefore, the numbers of catalytic waters should be considered as underestimates. $=$ Water has been proposed as a general catalyst in prebiotic chemistry (Saá and Frontera 2020).

Our attempt to quantify the number of water molecules involved in cellular metabolism is inspired in part by the general approach taken in Cell Biology by the Numbers (Milo and Phillips 2015).

\section{Transformations of Water During Protein Synthesis}

Protein constitutes about $52 \%$ of the dry weight of an E. coli (Milo 2013; Milo and Phillips 2015). We calculated the number of water molecules that are chemically involved in the biosynthesis of each of the 20 amino acids in an E. coli and weighted the results by relative amino acid abundance in $E$. coli. In addition, we determined the number of water molecules that chemically participate in polymerization of amino acids into protein.

Our calculations took the frequency of each amino acid in the E. coli $\mathrm{K}-12$ proteome into account, based on Proteome-pI database:

\begin{tabular}{lc}
\hline Amino acid & Frequency $(\%)$ \\
\hline Ala & 9.52 \\
Cys & 1.16 \\
Asp & 5.15 \\
Glu & 5.76 \\
Phe & 3.89 \\
Gly & 7.37 \\
His & 2.27 \\
Ile & 6.01 \\
Lys & 4.41 \\
Leu & 10.67 \\
Met & 2.82 \\
Asn & 3.95 \\
Gln & 4.44 \\
Pro & 4.43 \\
Arg & 5.51 \\
Ser & 5.8 \\
Thr & 5.4 \\
Val & 7.07 \\
Trp & 1.53 \\
Tyr & 2.85 \\
\hline
\end{tabular}

Source Kozlowski (2017)

The total number of amino acids in $E$. coli used here are obtained from Milo (2013) and Milo and Phillips (2015).

\begin{tabular}{ll}
\hline Number of proteins in an E. coli & $3.50 \times 10^{6}$ \\
Average number of AA/protein & $3.00 \times 10^{2}$ \\
Total number of AAs in an E. coli & $1.05 \times 10^{9}$ \\
\hline
\end{tabular}

Based on the relative frequency of the amino acids (AAs) in the E. coli proteome, the total number of proteinogenic amino acids incorporated into protein $\left(1.05 \times 10^{9}\right)$, the number of water molecules that are involved in synthesis of each of the 20 amino acids (Supporting Figs. S3, S4, S5, S6, S7, S8, S9, S10, S11, S12, S13, S14, S15, S16, S17, S18, S19, $\mathrm{S} 20, \mathrm{~S} 21, \mathrm{~S} 22)$, and the number of water molecules per $E$. coli $\left(2.34 \times 10^{10}\right)$, we calculated the percentage of water molecules that are involved in amino acid synthesis pathways.

Total number of an AA in protein $=[$ frequency of the AA in protein $\times$ total number of AAs incorporated into protein in an E. coli $\left(1.05 \times 10^{9}\right)$ ].

Total number of water molecules involved in the synthesis of an $\mathrm{AA}=$ total number of an $\mathrm{AA}$ in protein $\times$ number of water molecules involved in the synthesis of that AA. 
Percent of total water molecules in an E. coli involved in AA synthesis $=($ total number of water molecules involved in the synthesis of an AA $\times 100$ )/total number of water molecules involved in the synthesis of the AA by the total number of cellular water molecules $\left(2.34 \times 10^{10}\right)$.

The metabolic pathways for amino acid biosynthesis depicted here were based on the KEGG database for E. coli K-12 MG1655 strain (Kanehisa et al. 2007).

\begin{tabular}{|c|c|c|c|c|c|}
\hline $\begin{array}{l}\text { Amino } \\
\text { acid }\end{array}$ & $\begin{array}{l}\text { Fre- } \\
\text { quency } \\
\text { of AA in } \\
\text { pro- } \\
\text { teome } \\
(\%)\end{array}$ & $\begin{array}{l}\text { Total \# of } \\
\text { AAs }\end{array}$ & $\begin{array}{l}\text { Water } \\
\text { mol- } \\
\text { ecules/ } \\
\text { AA }\end{array}$ & $\begin{array}{l}\text { Total \# } \\
\text { of water } \\
\text { molecules }\end{array}$ & $\begin{array}{l}\text { Total \# of } \\
\text { water as } \\
\text { percent of } \\
\text { water in } E \text {. } \\
\operatorname{coli}(\%)\end{array}$ \\
\hline Ala & 9.52 & $1.00 \times 10^{8}$ & 6.5 & $6.50 \times 10^{8}$ & 2.78 \\
\hline Cys & 1.16 & $1.22 \times 10^{7}$ & 7.5 & $9.15 \times 10^{7}$ & 0.39 \\
\hline Asp & 5.15 & $5.41 \times 10^{7}$ & 19.5 & $1.05 \times 10^{9}$ & 4.51 \\
\hline Glu & 5.76 & $6.05 \times 10^{7}$ & 12.5 & $7.56 \times 10^{8}$ & 3.23 \\
\hline Phe & 3.89 & $4.08 \times 10^{7}$ & 15.5 & $6.32 \times 10^{8}$ & 2.70 \\
\hline Gly & 7.37 & $7.74 \times 10^{7}$ & 10.5 & $8.13 \times 10^{8}$ & 3.47 \\
\hline His & 2.27 & $2.38 \times 10^{7}$ & 15 & $3.57 \times 10^{8}$ & 1.53 \\
\hline Ile & 6.01 & $6.31 \times 10^{7}$ & 34 & $2.15 \times 10^{9}$ & 9.17 \\
\hline Lys & 4.41 & $4.63 \times 10^{7}$ & 36 & $1.67 \times 10^{9}$ & 7.12 \\
\hline Leu & 10.67 & $1.12 \times 10^{8}$ & 16 & $1.79 \times 10^{9}$ & 7.66 \\
\hline Met & 2.82 & $2.96 \times 10^{7}$ & 26.5 & $7.84 \times 10^{8}$ & 3.35 \\
\hline Asn & 3.95 & $4.15 \times 10^{7}$ & 21.5 & $8.92 \times 10^{8}$ & 3.81 \\
\hline Pro & 4.43 & $4.65 \times 10^{7}$ & 15.5 & $7.21 \times 10^{8}$ & 3.08 \\
\hline Gln & 4.44 & $4.66 \times 10^{7}$ & 12.5 & $5.83 \times 10^{8}$ & 2.49 \\
\hline Arg & 5.51 & $5.79 \times 10^{7}$ & 19.5 & $1.13 \times 10^{9}$ & 4.83 \\
\hline Ser & 5.8 & $6.09 \times 10^{7}$ & 6.5 & $3.96 \times 10^{8}$ & 1.69 \\
\hline Thr & 5.4 & $5.67 \times 10^{7}$ & 23.5 & $1.33 \times 10^{9}$ & 5.69 \\
\hline Val & 7.07 & $7.42 \times 10^{7}$ & 12 & $8.90 \times 10^{8}$ & 3.81 \\
\hline Trp & 1.53 & $1.61 \times 10^{7}$ & 18.5 & $2.98 \times 10^{8}$ & 1.27 \\
\hline Tyr & 2.85 & $2.99 \times 10^{7}$ & 14.5 & $4.34 \times 10^{8}$ & 1.85 \\
\hline Sum & & $1.05 \times 10^{9}$ & & $1.74 \times 10^{10}$ & 74.4 \\
\hline
\end{tabular}

Polymerization of amino acids into protein by the ribosome involves three water molecules per amino acid (Voet and Voet 2010): two of which are involved in GTP hydrolysis and one comes from hydrolysis of PPi following the amino acid adenylate formation.

In one E. coli there are $1.05 \times 10^{9}$ amide bonds (Milo 2013; Milo and Phillips 2015). The number of water molecules used for amino acid polymerization $=3$ (water molecules per polymerization of a single amino acid into protein) $\times 1.05 \times 10^{9}$ (total number of amide bonds in proteins in an $E$. coli $)=3.15 \times 10^{9}$ water molecules. An $E$. coli contains $2.34 \times 10^{10}$ water molecules (Milo and Phillips 2015), which is $3.89 \times 10^{-14} \mathrm{~mol}$.

For amino acid polymerization an E. coli uses $3.15 \times 10^{9}$ water molecules, which amounts to $13.5 \%$ of the total number of water molecules within the E. coli $\left[\left(3.15 \times 10^{9} / 2.34\right.\right.$ $\left.\times 10^{10}\right) \times 100$ ]. To sum up the amount of water molecules for synthesis of the amino acids and their subsequent polymerization, we add up the following processes. The $\%$ of $E$. coli water molecules used for protein synthesis $=\%$ water molecules used for amino acid synthesis $+\%$ water molecules for polymerization of amino acids $=74.4 \%+13.5 \%=87.9 \%$. If the doubling time of $E$. coli is $20 \mathrm{~min}$, then the average water molecule in $E$. coli is chemically transformed during protein synthesis $0.879 / 0.33=2.66$ times per hour. $E$. coli has $2.34 \times 10^{10}$ water molecules (Milo and Phillips 2015), which is $3.89 \times 10^{-14} \mathrm{~mol} .3 .42 \times 10^{-14} \mathrm{~mol}$ of water are utilized in protein synthesis $\left(=6.16 \times 10^{-13} \mathrm{~g}\right)$. Given a cell volume of $1 \times 10^{-15}(4)$, water molarity in an E. coli is $38.9 \mathrm{M}$.

\section{Water Transformations During Oxidative Phosphorylation}

Escherichia coli growing on glucose requires about $10 \times 10^{9}$ ATP molecules to replicate (Farmer and Jones 1976; Phillips and Milo 2009), which is $1.66 \times 10^{-14} \mathrm{~mol}$ of ATP. Under oxic conditions, E. coli invests in aerobic respiration over fermentation and anaerobic respiration. That is, 28 out of 32 molecules that are generated from a single glucose will be produced through oxidative phosphorylation $\left(=8.75 \times 10^{9}\right.$ ATP molecules) (Demirel 2013; Sokolov et al. 2015). The other 4 ATP molecules are generated through substrate-level phosphorylation.

As part of the oxidative phosphorylation pathway, the ATP synthase catalyzes dehydration of ADP and Pi into one ATP molecule (one water molecule is released during this process). In addition, ATP synthase has two mechanistic water molecules that are involved in each proton translocation across the membrane from the periplasm to the cytoplasm (Ribeiro et al. 2018). Since 3.3 protons are required for the generation of one ATP molecule by the ATP synthase (Voet and Voet 2010), and two water molecules are required mechanistically for the transport of each of those protons, then a total of about seven water molecules are required for the generation of one ATP molecule by the synthase. Given that $8.75 \times 10^{9}$ ATP molecules result from oxidative phosphorylation and that 7 water molecules per one ATP molecule are utilized by the ATP synthetase, then $6.125 \times 10^{10}$ water molecules are involved in oxidative phosphorylation in E. coli. Since E. coli has $2.34 \times 10^{10}$ water molecules, $262 \%$ of $E$. coli's water molecules are involved in this process of ATP production through aerobic respiration by the ATP synthase.

In addition, during oxidative phosphorylation there is delivery of electrons by the reduced NADH and $\mathrm{FADH}_{2}$ molecules to $\mathrm{O}_{2}$. The oxygen is being split to form water. Approximately $2 \mathrm{NADH}$ molecules result in the formation of 5 molecules of ATP and 2 water molecules (2 $\mathrm{NADH}=2 \mathrm{H}_{2} \mathrm{O}=5 \mathrm{ATP} \rightarrow \mathrm{ATP}=0.4 \mathrm{H}_{2} \mathrm{O}$ from $\mathrm{NADH}$ ) 
(51). Approximately 2 molecules of $\mathrm{FADH}_{2}$ result in the formation of 3 ATP molecules and 2 water molecules (2 $\mathrm{FADH}_{2}=2 \mathrm{H}_{2} \mathrm{O}=3 \mathrm{ATP} \rightarrow \mathrm{ATP}=0.66 \mathrm{H}_{2} \mathrm{O}$ from $\mathrm{FADH}{ }_{2}$ ) (Manoj et al. 2019). Out of the 28 ATP molecules that are generated per 1 glucose molecule through oxidative phosphorylation, approximately 26 ATP molecules will be generated through NADH, and 2 ATP molecules will be generated through $\mathrm{FADH}_{2}$. As $8.75 \times 10^{9}$ ATP molecules result from oxidative phosphorylation in E. coli, $8.125 \times 10^{9}$ $\left.\left[=(26 / 28) \times 8.75 \times 10^{9}\right)\right]$ are generated through NADH and $\left.0.625 \times 10^{9}\left[=(2 / 28) \times 8.75 \times 10^{9}\right)\right]$ are generated through $\mathrm{FADH}_{2}$. Such being the case, generation of ATP molecules through NADH will result in the transformation of $8.125 \times 10^{9} \times 0.4$ water molecules $\left(=3.25 \times 10^{9}\right)$, whereas generation of ATP molecules through $\mathrm{FADH}_{2}$ will result in the transformation of $0.625 \times 10^{9} \times 0.66$ water molecules $\left(=0.42 \times 10^{9}\right)$. Summed up together, generation of ATP molecules though splitting of oxygen mediated by NADH and $\mathrm{FADH}_{2}$ results in the formation of $3.67 \times 10^{9}$ water molecules. Since E. coli has $2.34 \times 10^{10}$ water molecules, $15.7 \%$ of $E$. coli water molecules are generated via splitting of oxygen.

To summarize, $278 \%$ of the water molecules of an $E$. coli are chemically transformed during oxidative phosphorylation. That is $6.50 \times 10^{10}$ molecules of water, or $1.08 \times 10^{-13} \mathrm{~mol}$, or $\left(=1.95 \times 10^{-12} \mathrm{~g}\right)$. If the doubling time of $E$. coli is $20 \mathrm{~min}$, then the average water molecule in $E$. coli is chemically transformed during oxidative phosphorylation $2.78 / 0.33=8.3$ times $/ \mathrm{h}$.

Acknowledgements We thank Drs. Charles L. Liotta, Elisa Biondi, George Rose, David Gutnick, Aikomarí Guzmán-Martínez, Steven A. Benner, Anthony Poole, Eric Smith, and Charles Lineweaver for helpful comments. This research was supported by the NSF and the NASA Astrobiology Program under the NSF Center for Chemical Evolution (CHE-1504217), the National Science Foundation (1724274), and by the NASA Astrobiology Program under the NASA Center for the Origins of Life (80NSSC18K1139). Moran Frenkel-Pinter acknowledges the NASA Astrobiology Postdoctoral Program.

Open Access This article is licensed under a Creative Commons Attribution 4.0 International License, which permits use, sharing, adaptation, distribution and reproduction in any medium or format, as long as you give appropriate credit to the original author(s) and the source, provide a link to the Creative Commons licence, and indicate if changes were made. The images or other third party material in this article are included in the article's Creative Commons licence, unless indicated otherwise in a credit line to the material. If material is not included in the article's Creative Commons licence and your intended use is not permitted by statutory regulation or exceeds the permitted use, you will need to obtain permission directly from the copyright holder. To view a copy of this licence, visit http://creativecommons.org/licenses/by/4.0/.

\section{References}

Anonymous (2010) M9 minimal medium (standard). Cold Spring Harbour Protocol

Apweiler R, Martin MJ, O'Donovan C, Magrane M, Alam-Faruque Y, Antunes R, Barrell D, Bely B, Bingley M, Binns D (2010) The universal protein resource (UniProt) in 2010. Nucleic Acids Res 38:D142-D148

Ball P (2017) Water is an active matrix of life for cell and molecular biology. Proc Natl Acad Sci USA 114:13327-13335

Barron LD, Hecht L, Wilson G (1997) The lubricant of life: a proposal that solvent water promotes extremely fast conformational fluctuations in mobile heteropolypeptide structure. Biochemistry 36:13143-13147

Belogurov GA, Artsimovitch I (2015) Regulation of transcript elongation. Annu Rev Microbiol 69:49-69

Bennett BD, Kimball EH, Gao M, Osterhout R, Van Dien SJ, Rabinowitz JD (2009) Absolute metabolite concentrations and implied enzyme active site occupancy in Escherichia coli. Nat Chem Biol 5:593-599

Bowman JC, Lenz TK, Hud NV, Williams LD (2012) Cations in charge: magnesium ions in RNA folding and catalysis. Curr Opin Struct Biol 22:262-272

Brack A (1993) Liquid water and the origin of life. Orig Life Evol Biosph 23:3-10

Buttgereit F, Brand MD (1995) A hierarchy of ATP-consuming processes in mammalian cells. Biochem J 312:163-167

Capaldi RA, Aggeler R (2002) Mechanism of the F1F0-type ATP synthase, a biological rotary motor. Trends Biochem Sci 27:154-160

Demirel Y (2013) Nonequilibrium thermodynamics: transport and rate processes in physical, chemical and biological systems. Elsevier, New York

Doran D, Abul-Haija YM, Cronin L (2019) Emergence of function and selection from recursively programmed polymerisation reactions in mineral environments. Angew Chem Int Ed 58:11253-11256

Farmer IS, Jones CW (1976) The energetics of Escherichia coli during aerobic growth in continuous culture. Eur J Biochem 67:115-122

Fell DA, Wagner A (2000) The small world of metabolism. Nat Biotechnol 18:1121-1122

Forsythe JG, Yu SS, Mamajanov I, Grover MA, Krishnamurthy R, Fernández FM, Hud NV (2015) Ester-mediated amide bond formation driven by wet-dry cycles: a possible path to polypeptides on the prebiotic earth. Angew Chem Int Ed 54:9871-9875

Frenkel-Pinter M, Haynes JW, Martin C, Petrov AS, Burcar BT, Krishnamurthy R, Hud NV, Leman LJ, Williams LD (2019) Selective incorporation of proteinaceous over nonproteinaceous cationic amino acids in model prebiotic oligomerization reactions. Proc Natl Acad Sci USA 116:16338-16346

Hahn A, Parey K, Bublitz M, Mills DJ, Zickermann V, Vonck J, Kühlbrandt W, Meier T (2016) Structure of a complete ATP synthase dimer reveals the molecular basis of inner mitochondrial membrane morphology. Mol Cell 63:445-456

Hertzog J, Naraoka H, Schmitt-Kopplin P (2019) Profiling Murchison soluble organic matter for new organic compounds with APPI-and ESI-FT-ICR MS. Life 9:48

Ingledew W, Poole R (1984) The respiratory chains of Escherichia coli. Microbiol Rev 48:222

Kanehisa M, Araki M, Goto S, Hattori M, Hirakawa M, Itoh M, Katayama T, Kawashima S, Okuda S, Tokimatsu T (2007) KEGG for linking genomes to life and the environment. Nucleic Acids Res 36:D480-D484

Kauzmann W (1959) Some factors in the interpretation of protein denaturation. Adv Protein Chem 14:1-63 
Kim P-J, Lee D-Y, Kim TY, Lee KH, Jeong H, Lee SY, Park S (2007) Metabolite essentiality elucidates robustness of Escherichia coli metabolism. Proc Natl Acad Sci USA 104:13638-13642

Kozlowski LP (2017) Proteome-pi: proteome isoelectric point database. Nucleic Acids Res 45:D1112-D1116

Li A (2020) Spitzer's perspective of polycyclic aromatic hydrocarbons in galaxies. Nat Astron 4:339-351

Mamajanov IM, Macdonald PJ, Ying J, Duncanson DM, Dowdy GR, Walker CA, Engelhart AE, Fernández FM, Grover MA, Hud NV, Schork FJ (2014) Ester formation and hydrolysis during wet-dry cycles: generation of far-from-equilibrium polymers in a model prebiotic reaction. Macromolecules 47:1334-1343

Manoj KM, Soman V, Jacob VD, Parashar A, Gideon DA, Kumar M, Manekkathodi A, Ramasamy S, Pakshirajan K, Bazhin NM (2019) Chemiosmotic and Murburn explanations for aerobic respiration: predictive capabilities, structure-function correlations and chemico-physical logic. Arch Biochem Biophys 676:108128

Mellanby K (1942) Metabolic water and desiccation. Nature 150:21-21

Milo R (2013) What is the total number of protein molecules per cell volume? A call to rethink some published values. BioEssays 35:1050-1055

Milo R, Phillips R (2015) Cell biology by the numbers. Garland Science, New York

Moradi P, Ford-Lloyd B, Pritchard J (2017) Comprehensive list of metabolites measured by DI-FTICR mass spectrometry in thyme plants with contrasting tolerance to drought. Data Brief 12:438-441

Nagano N, Nakayama N, Ikeda K, Fukuie M, Yokota K, Doi T, Kato T, Tomii K (2015) EzCatDB: the enzyme reaction database, 2015 update. Nucleic Acids Res 43:D453-D458

Patel SS, Pandey M, Nandakumar D (2011) Dynamic coupling between the motors of DNA replication: hexameric helicase, DNA polymerase, and primase. Curr Opin Chem Biol 15:595-605

Phillips R, Milo R (2009) A feeling for the numbers in biology. Proc Natl Acad Sci USA 106:21465-21471

Radzicka A, Pedersen L, Wolfenden R (1988) Influences of solvent water on protein folding: free energies of solvation of $c i s$ and trans peptides are nearly identical. Biochemistry 27:4538-4541

Ribeiro AJM, Holliday GL, Furnham N, Tyzack JD, Ferris K, Thornton JM (2018) Mechanism and catalytic site atlas (M-CSA): a database of enzyme reaction mechanisms and active sites. Nucleic Acids Res 46:D618-D623
Ross DS, Deamer D (2016) Dry/wet cycling and the thermodynamics and kinetics of prebiotic polymer synthesis. Life 6:28

Runnels CM, Lanier KA, Williams JK, Bowman JC, Petrov AS, Hud NV, Williams LD (2018) Folding, assembly, and persistence: the essential nature and origins of biopolymers. J Mol Evol 86:598-610

Russell JB, Cook GM (1995) Energetics of bacterial growth: balance of anabolic and catabolic reactions. Microbiol Mol Biol Rev 59:48-62

Saá JM, Frontera A (2020) On the role of water as a catalyst in prebiotic chemistry. ChemPhysChem 21:313-320

Sokolov S, Balakireva A, Markova O, Severin F (2015) Negative feedback of glycolysis and oxidative phosphorylation: mechanisms of and reasons for it. Biochemistry (Mosc) 80:559-564

Stouthamer AH (1973) A theoretical study on the amount of ATP required for synthesis of microbial cell material. Antonie Van Leeuwenhoek 39:545-565

Sundaralingam M, Sekharudu YC (1989) Water-inserted alpha-helical segments implicate reverse turns as folding intermediates. Science 244:1333-1337

Szent-Györgyi A (1971) Biology and pathology of water. Perspect Biol Med 14:239-249

Testa B, Kraemer SD (2007) The biochemistry of drug metabolisman introduction: Part 3. Reactions of hydrolysis and their enzymes. Chem Biodivers 4:2031-2122

Tran QH, Unden G (1998) Changes in the proton potential and the cellular energetics of Escherichia coli during growth by aerobic and anaerobic respiration or by fermentation. Eur J Biochem 251:538-543

Venes D (ed) (1940) Taber's cyclopedic medical dictionary. F.A. Davis Company, Philadelphia

Voet D, Voet J (2010) Biochemistry, 4th edn. Wiley, New York

Weingärtner H, Franck EU, Wiegand G, Dahmen N, Schwedt G, Frimmel FH, Gordalla BC (2000) Water, 1. Properties, analysis and hydrological cycle. In: Ullmann's encyclopedia of industrial chemistry. Wiley-VCH Verlag GmbH \& Co. KGaA, Weinheim

Wishart DS, Feunang YD, Marcu A, Guo AC, Liang K, VázquezFresno R, Sajed T, Johnson D, Li C, Karu N (2018) HMDB 4.0: the human metabolome database for 2018. Nucleic Acids Res 46:D608-D617 